\title{
Kidney disease among children in sub-Saharan Africa: systematic review
}

\author{
Neema M. Kayange ${ }^{1}$, Luke R. Smart ${ }^{2,3}$, Jacob E. Tallman ${ }^{4}$, Emily Y. Chu ${ }^{4}$, Daniel W. Fitzgerald ${ }^{3}$, Kevin J. Pain ${ }^{5}$ and Robert N. Peck ${ }^{2,3}$
}

The global burden of kidney disease is increasing, and several etiologies first begin in childhood. Risk factors for pediatric kidney disease are common in Africa, but data regarding its prevalence are lacking. We completed a systematic review of community-based studies describing the prevalence of proteinuria, hematuria, abnormal imaging, or kidney dysfunction among children in sub-Saharan Africa (SSA). Medline and Embase were searched. Five hundred twenty-three references were reviewed. Thirty-two references from nine countries in SSA were included in the qualitative synthesis. The degree of kidney damage and abnormal imaging varied widely: proteinuria 32.5\% (2.2-56.0\%), hematuria 31.1\% (0.6-67.0\%), hydronephrosis $11.3 \%$ (0.0-38.0\%), hydroureter $7.5 \%$ (0.0-26.4\%), and major kidney abnormalities $0.1 \%(0.0-0.8 \%)$. Serum creatinine was reported in four studies with insufficient detail to identify the prevalence renal dysfunction. A majority of the studies were performed in Schistosoma haematobium endemic areas. A lower prevalence of kidney disease was observed in the few studies from nonendemic areas. Published data on pediatric kidney disease in SSA are highly variable and dependent on S. haematobium prevalence. More community-based studies are needed to describe the burden of pediatric kidney disease, particularly in regions where $S$. haematobium infection is nonendemic.

W orldwide, the number of deaths attributable to chronic kidney disease (CKD) has almost doubled in the past 20 years, ranking it among the top 20 causes of death globally (1). Along with other noncommunicable diseases, CKD has begun to gain recognition as an important contributor to the burden of disease not only in high-income countries, but also in low-income countries in regions such as sub-Saharan Africa (SSA). Although high quality epidemiological data from SSA are sparse, available published data suggest the prevalence of CKD is substantial (2).

Pediatric registries in several high-income countries (3-5) have reported that the incidence of pediatric CKD is relatively low in such settings and that congenital anomalies of the kidney and urinary tract cause $50 \%$ or more of CKD in children. In studies from the United States, the incidence of CKD is two to three times higher among African American children than among Caucasian children. This is partly due to the higher frequency of primary glomerular diseases such as focal segmental glomerular sclerosis (3). This difference may be related to the APOL1 gene mutation $(6,7)$, which may also play a role in kidney disease in SSA (8).

Risk factors for CKD are more common among children in SSA. Intrauterine injury to the developing kidney and malnutrition in childhood are more common in low-income populations and contribute to the increased prevalence of $\mathrm{CKD}$ $(9,10)$. Parasitic infections such as schistosomiasis, postinfectious glomerulonephritis, HIV-related nephropathy, and sickle disease all cause damage to the kidney in childhood and are more common in SSA (11-14). In addition, acute kidney disease is more common among children in SSA, particularly due to higher rates of diarrhea, and may progress to CKD (15-17).

Despite these risk factors, data regarding pediatric CKD in SSA remain sparse and are mostly limited to health facilitybased studies. Therefore, we completed a systematic review of studies investigating kidney disease among community-dwelling children in SSA.

\section{RESULTS}

\section{Search Results}

See Figure 1 for a Preferred Reporting Items for Systematic Reviews and Meta-Analyses diagram explaining the search results and exclusion process. We identified 688 references through database searching. An additional 45 references were found through hand searching. After removal of duplicates, 523 unique references remained. Upon review of titles and abstracts, 432 references were excluded, and 91 references remained for full text review. An additional 59 references were excluded upon full-text review, leaving 32 references that met our inclusion and exclusion criteria. After merging duplicate data sets and identifying distinct data sets, a total of 35 data sets were taken from these 32 references (18-49) for qualitative synthesis.

\footnotetext{
The first two authors contributed equally to this work.

'Department of Pediatrics, Weill Bugando School of Medicine, Catholic University of Health and Allied Sciences, Mwanza, Tanzania; ${ }^{2}$ Department of Internal Medicine, Weill Bugando School of Medicine, Catholic University of Health and Allied Sciences, Mwanza, Tanzania; ${ }^{3}$ Department of Medicine, Center for Global Health, Weill Cornell Medical College, New York, New York; ${ }^{4}$ Cornell University, Ithaca, New York; ${ }^{5}$ Samuel J. Wood Library/CV Starr Biomedical Information Center, Weill Cornell Medical College, New York, New York. Correspondence: Luke R. Smart (smart.luke@gmail.com)
} 


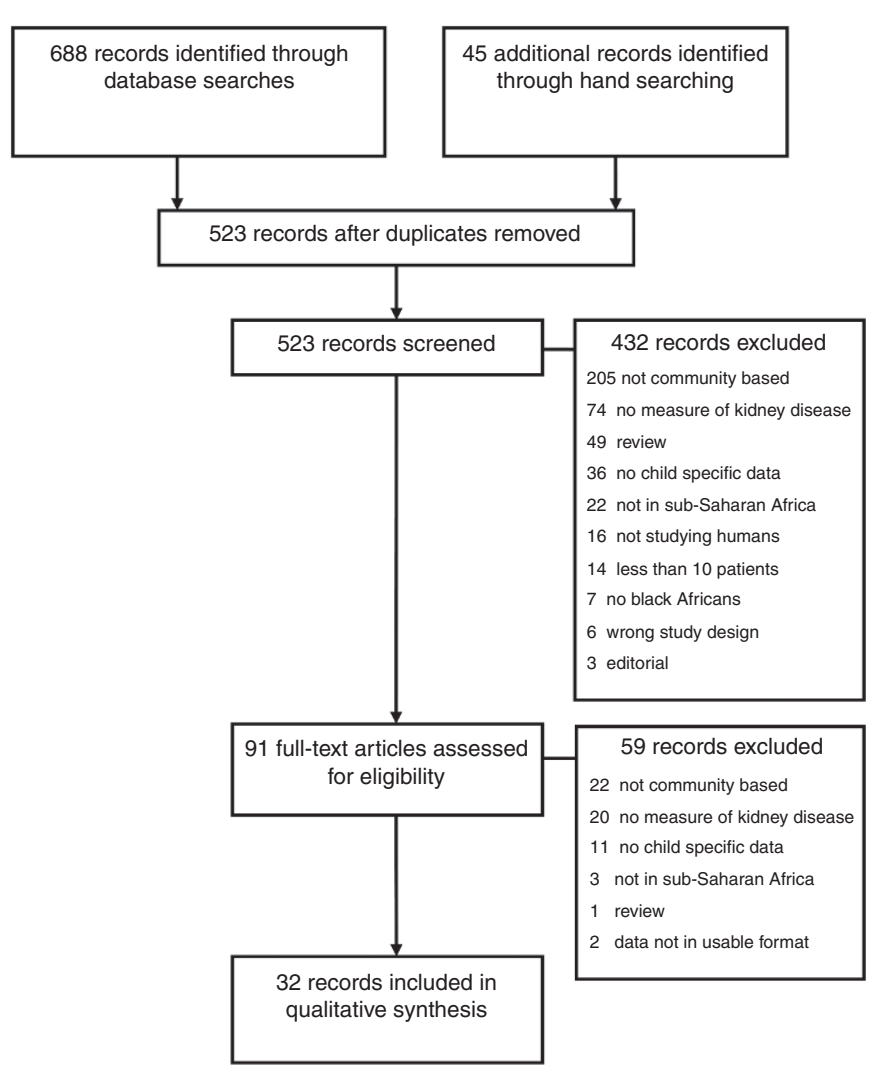

Figure 1. Preferred Reporting Items for Systematic Reviews and Meta-Analyses (PRISMA) diagram of study selection process.

\section{Study Characteristics}

See Table 1 for characteristics of included data sets. The data represented nine countries in SSA (Benin, Cameroon, Kenya, Liberia, Mali, Niger, Nigeria, Tanzania, and Zimbabwe), and was published between 1964 and 2012. The age range of subjects was $0-21$ y old (average age 8.7-13.6). Female subjects represented $0-55 \%$. Home-based sampling was done in 12 instances and school-based sampling in 23. Sample size was from 40 to 2,628 subjects. The risk of selection bias score ranged from 1 to 2 (good to moderate). The risk of measurement bias score ranged from 2 to 3 (moderate to poor).

Several of the studies were carried out as part of multiyear projects. Often these studies described the prevalence of S. haematobium, or the effects of treatment for schistosomiasis. Examples include the Msambweni project in Kenya from 1984 to 1992 (31-34,36,38), Malumfashi Endemic Research Project and other sites in Nigeria $(25,44)$, and several studies in both Tanzania $(20,22,23)$ and Mali $(29,47,48)$.

\section{Proteinuria}

See Table 2 for the reported prevalence of proteinuria in included data sets. The prevalence of proteinuria was 2.2$56.0 \%$ (average $32.5 \%$ ). In the majority of data sets, the only identified factor associated with proteinuria was the presence and/or intensity of $S$. haematobium infection. Only four studies investigated populations without $S$. haematobium $(28,39-$ 42). Two of these did not find any factors associated with proteinuria $(39,42)$. One found that proteinuria was associated with malaria infection $(P<0.05)(28)$. The last found that microalbuminuria was associated with hypertension (odds ratio $(\mathrm{OR})=5.4 ; 95 \% \mathrm{CI}=1.8-16.2 ; P=0.001)(40,41)$.

The prevalence of proteinuria varied considerably even in these populations unaffected by S. haematobium. The two lowest outliers reported proteinuria in 2.2 and $4.7 \%$ of urban secondary school students from Nairobi, Kenya (39) and Benin City, Nigeria (42), respectively. In contrast, $49.8 \%$ of students in Port Harcourt City, Nigeria had proteinuria (23.7\% with macroalbuminuria and $26.1 \%$ with microalbuminuria). A fourth study found pathologic proteinuria in $18.2 \%$ of rural primary school children in Kenya who had a high prevalence of S. mansoni (91.9\% infected) (28). The cut off for pathologic proteinuria in this study was $200 \mathrm{mg} / \mathrm{l}$ (as opposed to the $30 \mathrm{mg} / \mathrm{l}$ that most other studies used), suggesting that the number of students with proteinuria was likely much higher.

\section{Hematuria}

See Table 2 for details of reported prevalence of hematuria in included data sets. The prevalence of hematuria varied widely from 0.6 to $67.0 \%$ (average $31.1 \%$ ). In the majority of data sets, the only identified factor associated with hematuria was the presence and/or intensity of S. haematobium infection. Other associated factors were female sex, sickle cell anemia, and glomerulonephritis $(18,42)$. The three studies with lowest prevalence $(0.6-3.5 \%)$ were conducted in urban populations that were not tested for S. haematobium (39-42). None of these identified any factors associated with hematuria. The next lowest prevalence (5.4\%) was identified in rural primary school children (18) with a low prevalence of schistosomiasis (5.2\%), but 46 of the 48 subjects with hematuria (96\%) were infected with S. haematobium.

\section{Serum Creatinine}

See Table 3 for details of reported serum creatinine (SCr) in included data sets. No studies calculated an estimated glomerular filtration rate (eGFR). Only one study from Kenya tested SCr in all subjects (28). They reported that all of the children had normal values, but they did not specify a reference range or report a median, mean, or range of creatinine values.

Three other studies reported SCr values for Nigerian children. One stated that children with hematuria or proteinuria had an SCr $<115 \mu \mathrm{mol} / \mathrm{l}$ both at enrollment and at follow up 1 y later (42). Another reported a mean SCr of $83.3 \mu \mathrm{mol} / 1$ (range 71-108) in 46 subjects infected with $S$. haematobium (18). The last one found an SCr range of 30-82 $\mu \mathrm{mol} / \mathrm{l}$ in 69 male subjects (age 7-17) who had a high intensity schistosomiasis infection (44). None of these studies made any attempt to adjust their range of normal to accommodate the weight, nutritional status, or age of the patient.

\section{Kidney or Ureter Imaging}

See Table 4 for details of reported imaging abnormalities in included data sets. The prevalence of any imaging abnormality was $0.5-38.0 \%$ (average 14.8\%), and except for five studies, all 
Table 1. Characteristics of included studies

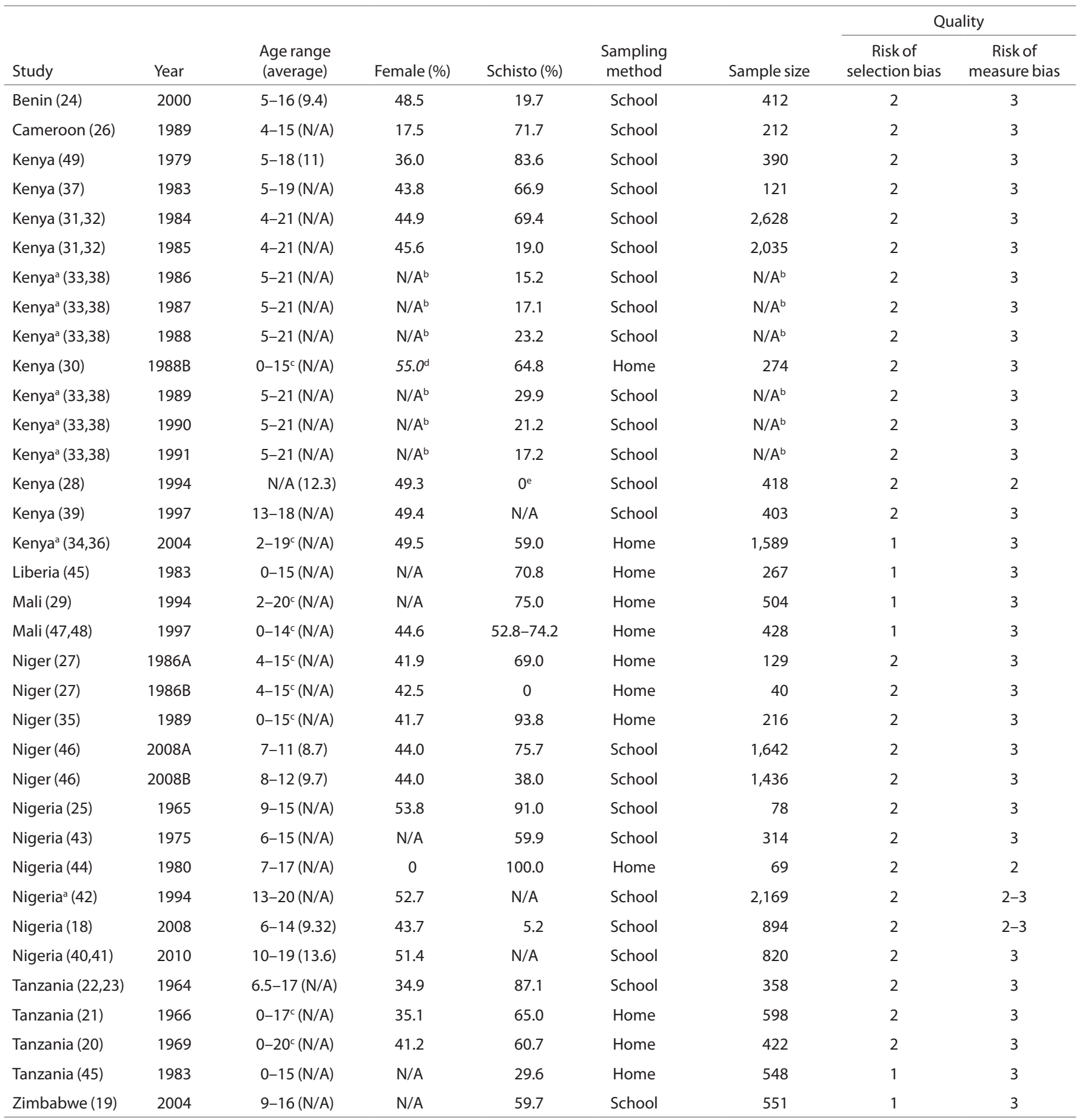

IVP, intravenous pyelogram; N/A, not available; Schisto, schistosomiasis.

a Data extracted from figures in article using ImageJ software (US National Institutes of Health). bFollow up study in the Msambweni project. 'Original study included adults. Data reported in this table are only for pediatric patients $<21 \mathrm{y}$ old. dData provided in italics are for the whole study population including adults. These statistics are not provided for the pediatric subpopulation. Total population and female ratio not reported in summary articles. ${ }^{\text {This }}$ population was endemic for S. mansoni rather than S. haematobium with a $91.9 \%$ prevalence of S. mansoni.

reported a prevalence $>10.0 \%$. The prevalence of hydronephrosis was $0-38.0 \%$ (average $11.3 \%$ ), the prevalence of hydroureter was $0-26.4 \%$ (average $7.5 \%$ ), and the prevalence of major kidney abnormalities was $0-0.8 \%$ (average $0.1 \%$ ).

Studies used either intravenous pyelogram or ultrasound (USS). Intravenous pyelogram was completed in hospitals or clinics. USS was usually completed in the field. Five of the USS data sets stated that a radiologist performed the test. In other studies, the investigators performed the USS. None of the studies described the training of USS technicians or operators. Abnormal findings were reported differently in the various studies, but abnormalities invariably fell into one of three 


\section{Pediatric kidney disease in Africa $\quad$ Systematic Review}

Table 2. Urine proteinuria and hematuria from included studies

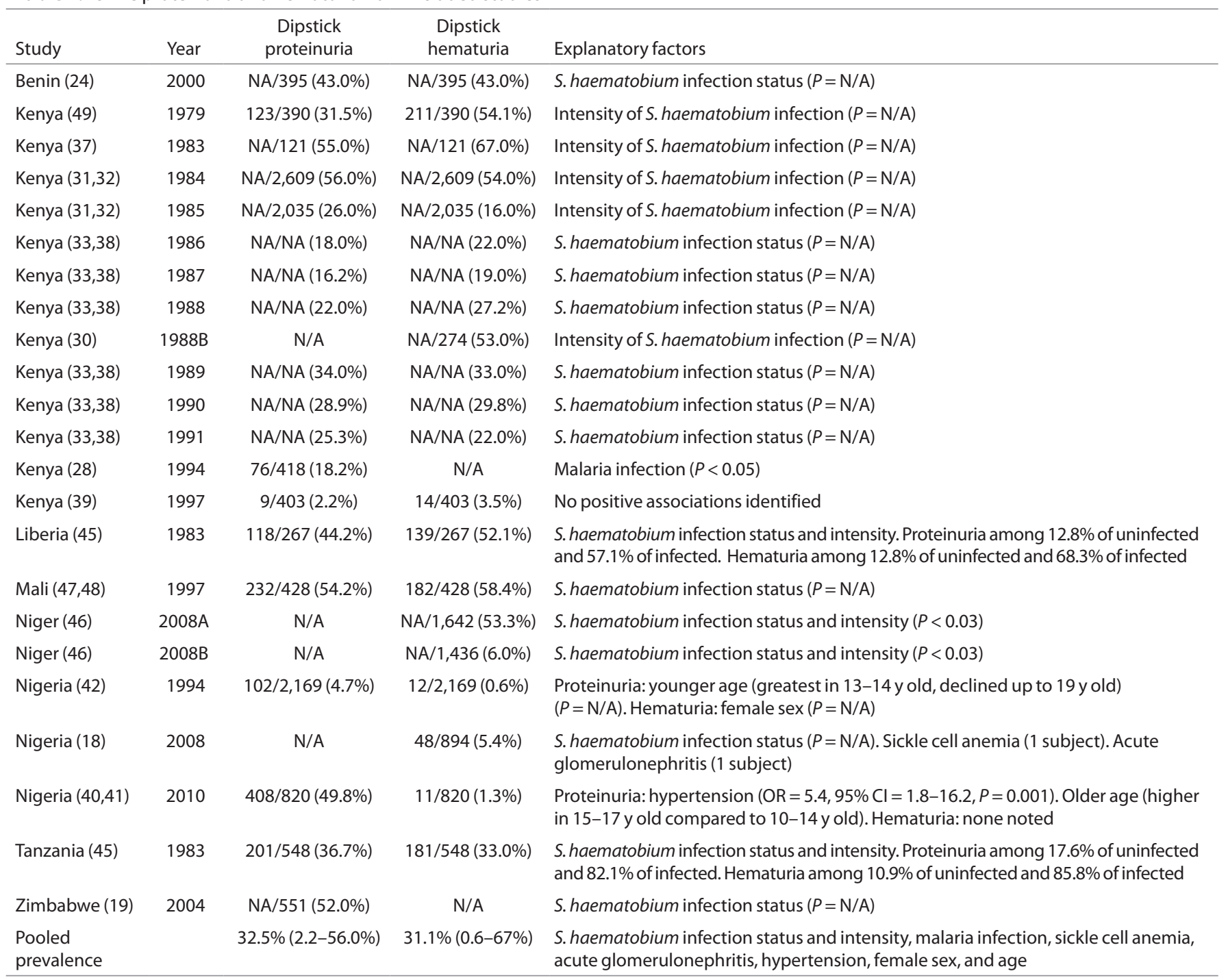

$\mathrm{Cl}$, confidence interval; $\mathrm{N} / \mathrm{A}$, not available; $\mathrm{OR}$, odds ratio.

categories: hydronephrosis, hydroureter, or major kidney abnormality. All imaging studies were completed in populations endemic for $S$. haematobium. In 12 of the data sets, all subjects underwent imaging, whereas only a randomly selected subgroup of study subjects underwent imaging in the other 6 data sets.

\section{Association With S. haematobium Infection}

See Table 5 for details of the relationship between S. haematobium prevalence and kidney disease. The majority of the studies investigating pediatric kidney disease in SSA were performed in areas with a high prevalence of $S$. haematobium (15.0-93.8\%). Two studies had a prevalence of $0-5 \%$ $(18,27)$, and three did not record the prevalence of S. haematobium (39-42). In Niger, investigators found significantly more hydronephrosis $(38.0 \%)$ in an area with a high prevalence of S. haematobium (69.0\%) compared to the amount of hydronephrosis (12.5\%) in an area without any $S$. haematobium (27). Other studies documented the decrease in prevalence of kidney disease after treatment for $S$. haematobium $(31-33,38,46)$.

For data sets that reported proteinuria, there was not a clearly defined association between schistosomiasis prevalence and prevalence of proteinuria, but an increasing $S$. haematobium endemicity was generally associated with a higher prevalence of proteinuria as seen in Table 5. Two notable outliers from this trend were Okpere who found a $49.8 \%$ prevalence of proteinuria in a population that was not endemic for schistosomiasis $(40,41)$ and Warren who found a $31.5 \%$ prevalence of proteinuria despite a $83.6 \%$ prevalence of $S$. haematobium (49).

All studies that investigated the association between proteinuria and S. haematobium infection found that S. haematobium status and/or intensity were associated with proteinuria. For example, in the first year of the Msambweni project in Kenya $(31,32)$, the prevalence of proteinuria was $15.0 \%$ among uninfected schoolchildren and $74.0 \%$ among infected children. Moreover, when students were treated for schistosomiasis, the prevalence of proteinuria decreased in the overall study 


\section{Systematic Review | Kayange etal.}

Table 3. Serum creatinine data from included studies

\begin{tabular}{|c|c|c|}
\hline Study & Year & Creatinine $(\mu \mathrm{mol} / \mathrm{l})$ \\
\hline Kenya (28) & 1994 & $\begin{array}{l}\text { "All values were found to } \\
\text { be within normal range" }\end{array}$ \\
\hline Nigeria (44) & 1980 & Range $30-82^{b}$ \\
\hline Nigeria (42) & 1994 & All measurements $<115^{c}$ \\
\hline Nigeria (18) & 2008 & Range 71-108 (mean 83.3) ${ }^{d}$ \\
\hline \multicolumn{3}{|c|}{$\begin{array}{l}\text { aDirect quote from study manuscript. No median, mean, or range provided in study } \\
\text { manuscript. No specification of normal reference range provided. }{ }^{b} \text { Only tested in } 69 \\
\text { boys ( } 7-17 \text { y old) selected for intense schistosomiasis infection. No median or mean } \\
\text { provided in study manuscript. Normal reference range was stated as } 9-124 \mu \mathrm{mol} / \mathrm{l} \text {. } \\
\text { cOnly tested in those with previously identified kidney abnormalities (e.g., hematuria or } \\
\text { proteinuria). Serum creatinine was measured both at the beginning of the study and } \\
\text { after } 1 \text { y of follow up. No median, mean, or range provided in study manuscript. Normal } \\
\text { reference range was stated as }<115 \mu \text { mol/l. }{ }^{~} \text { Only tested in those with schistosomiasis } \\
\text { infection. Normal reference range was stated as } 50-110 \mu \mathrm{mol} / \mathrm{l} \text {. }\end{array}$} \\
\hline
\end{tabular}

population the following year (from $56.0 \%$ before treatment to $26.0 \%$ after treatment) along with the prevalence of schistosomiasis (from $69.4 \%$ before treatment to $19.0 \%$ after treatment).

For studies of hematuria, a similar association with $S$. haematobium prevalence was observed. All studies that investigated hematuria and S. haematobium found an association between the two. Again, the Msambweni project is a case in point (32). The prevalence of hematuria was $6.0 \%$ among uninfected subjects and $76.0 \%$ among infected subjects. One year after treatment, the population prevalence of hematuria in the overall population decreased (from $54.0 \%$ before treatment to $16.0 \%$ after treatment) along with the prevalence of schistosomiasis (from $69.4 \%$ before treatment to $19.0 \%$ after treatment) $(31,32)$. Tohon also observed this phenomenon. One year after treatment for S. haematobium, the prevalence of hematuria decreased (from $60.2 \%$ before treatment to $6.4 \%$ after treatment) along with the $S$. haematobium prevalence (from $75.7 \%$ before treatment to $38.0 \%$ after treatment) (46).

In imaging studies, the association between S. haematobium infection and imaging abnormalities was not as consistent. Some studies showed a high prevalence of $S$. haematobium $(19.7,38.0,59.0$, and $75.7 \%)$ but a very low prevalence of abnormal imaging $(0.5,0.6,0.9$, and $4.2 \%)(24,34,36,46)$. One study (35) found no association between S. haematobium infection and imaging abnormalities at all. This was in contrast to 17 other data sets which identified an association between imaging abnormalities and S. haematobium infection. Improvement in imaging abnormalities after treatment was also variable, with some studies demonstrating variable improvement in imaging (20) and others failing to show improvement $(31,32)$.

Only one study examined the relationship between $S$. mansoni infection and kidney disease (28). The population studied had a very high prevalence of $S$. mansoni $(91.9 \%)$ and no $S$. haematobium (0\%). They found pathologic proteinuria $(\geq 200 \mathrm{mg} / \mathrm{l})$ in $18.2 \%$ of the children, and a normal SCr in all children. Despite setting their limit for pathologic proteinuria higher than other studies $(\geq 200 \mathrm{mg} / \mathrm{l}$ rather than $30 \mathrm{mg} / \mathrm{l})$, they still identified a significant amount of kidney disease. They reported no association between S. mansoni infection intensity and kidney function or proteinuria in these subjects.
However, the prevalence of $S$. mansoni was so high that the power to detect pathologic differences between children with and without $S$. mansoni infection was very low.

\section{DISCUSSION}

Markers of kidney disease are very common among children living in of SSA. In the community-based studies that we reviewed, average proteinuria prevalence was $32.5 \%(2.2-56.0 \%)$, average hematuria prevalence was $31.1 \%(0.6-67 \%)$, and imaging abnormalities were found in $14.8 \%(0.5-38.0 \%)$. This is higher than the degree of damage identified in adults both in the United States (50) and SSA (2). It is much higher than the amount of disease identified in children in the United States (51-53). Data from the US National Health and Nutritional Examination Survey identified microalbuminuria in $9.9 \%$ of children between 6 and 19 y old (52). In a larger study of school children in the United States, Dodge et al. found that cumulative proteinuria and hematuria amounted to only $6.0 \%$ (54).

Proteinuria, in particular, seems to be common among African children. Except for two lower outliers, proteinuria was found in $16.2-56.0 \%$ of children. Unfortunately, none of the studies used the gold-standard measurement of albumincreatinine ratio to diagnose albuminuria. This amount of proteinuria is substantially more than the amount of proteinuria in children (9.9\%) and adults (9.2\%) from the United States $(50,52)$ and is also higher than adults from SSA $(6-24 \%)(2)$. Albuminuria in adults is known to increase a patient's risk of cardiovascular disease and mortality (55), but these associations have primarily been observed in high-income countries. The long-term implications of pediatric albuminuria are still unclear in high-income countries, and the significance of such high levels of proteinuria in children from SSA is even more obscure. Specifically, we do not know if community dwelling children in SSA with proteinuria will progress to end stage renal disease or whether it will lead to increased risk of cardiovascular disease and death. Prospective long-term cohort studies are needed to define the outcomes of these patients.

Genitourinary imaging abnormalities were common among included studies. All but five studies had imaging abnormalities that were greater than $10 \%$. The degree of hydronephrosis and hydroureter in particular were very high in some studies. Some of these abnormalities were reversible after treatment for schistosomiasis, but many were not. While the number of major abnormalities (nonfunctioning or missing kidney) was very low $(<1 \%)$, a high prevalence of hydronephrosis is worrisome. The effects of obstructive uropathy in high-income countries are more often observed in young children from anatomic congenital disease of the urinary tract or elderly males from prostatic hypertrophy, but chronic partial obstruction of the ureter from any cause will result in deterioration of renal function $(56,57)$. The ongoing damage caused to the kidney by the obstruction involves a complex interplay of physics and molecular inflammatory signaling that can lead to progressive kidney disease, especially when the damage has been acquired in childhood (58). Screening renal USS and regular follow up of renal function and obstructive pathology in SSA, especially 


\section{Pediatric kidney disease in Africa $\quad$ Systematic Review}

Table 4. Imaging data from included studies

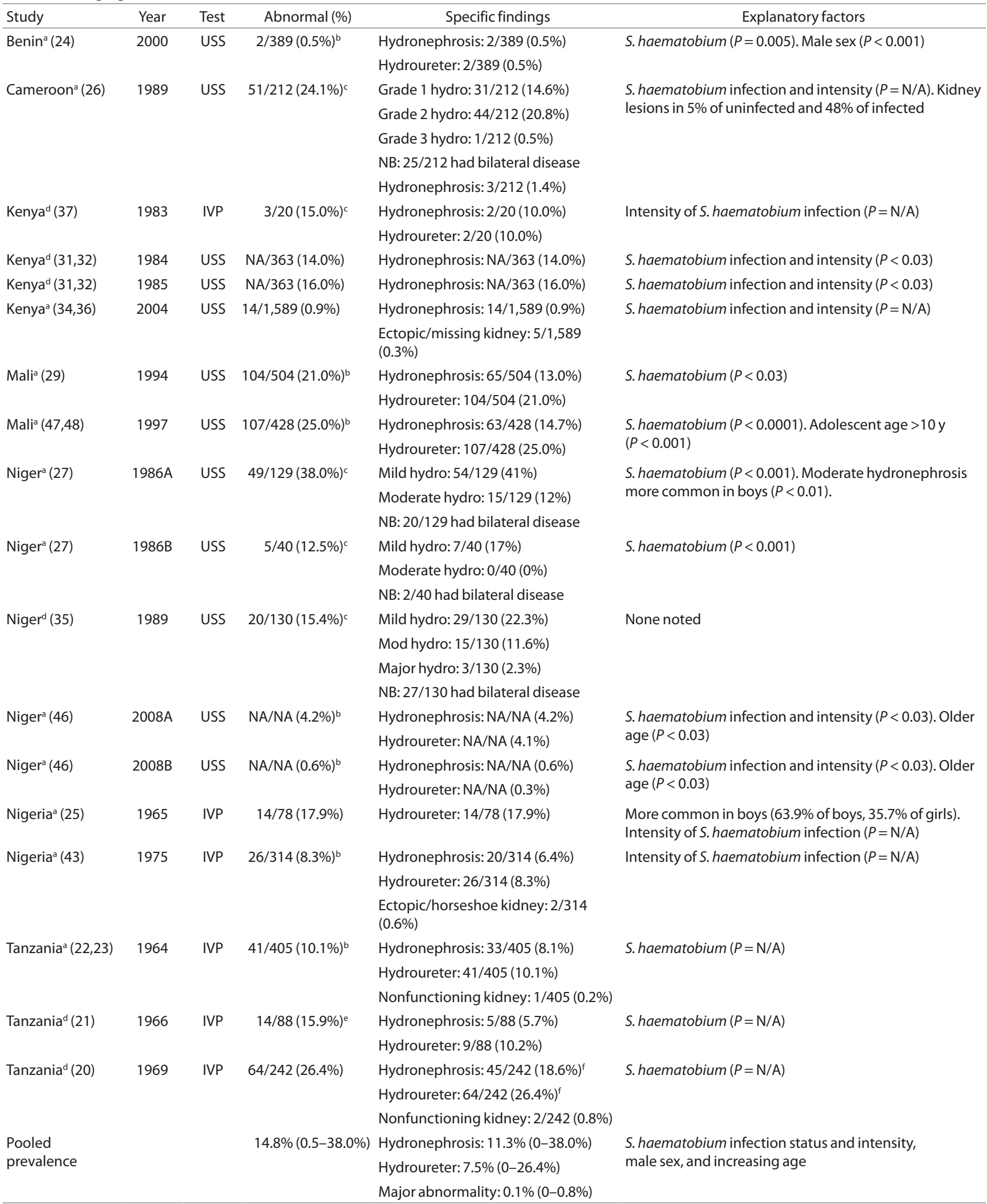

$\mathrm{Cl}$, confidence interval; IVP, intravenous pyelogram; N/A, not available; NB, nota bene; OR, odds ratio; USS, ultrasound.

almaging performed on whole study population. ${ }^{b}$ No explicit mention of whether lesions cooccur, therefore the greatest number of single lesions was taken as a conservative estimate of the prevalence of abnormal imaging to avoid overestimation. Total prevalence of imaging abnormalities lower than cumulative number of individual abnormalities because of children with bilateral abnormalities. IImaging performed on random sample of study population. 'Lack of cooccurrence clearly stated. 'Bilateral lesions in the same patient counted separately resulting in overestimate of prevalence. 
Table 5. Relationship between S. haematobium and markers of renal disease

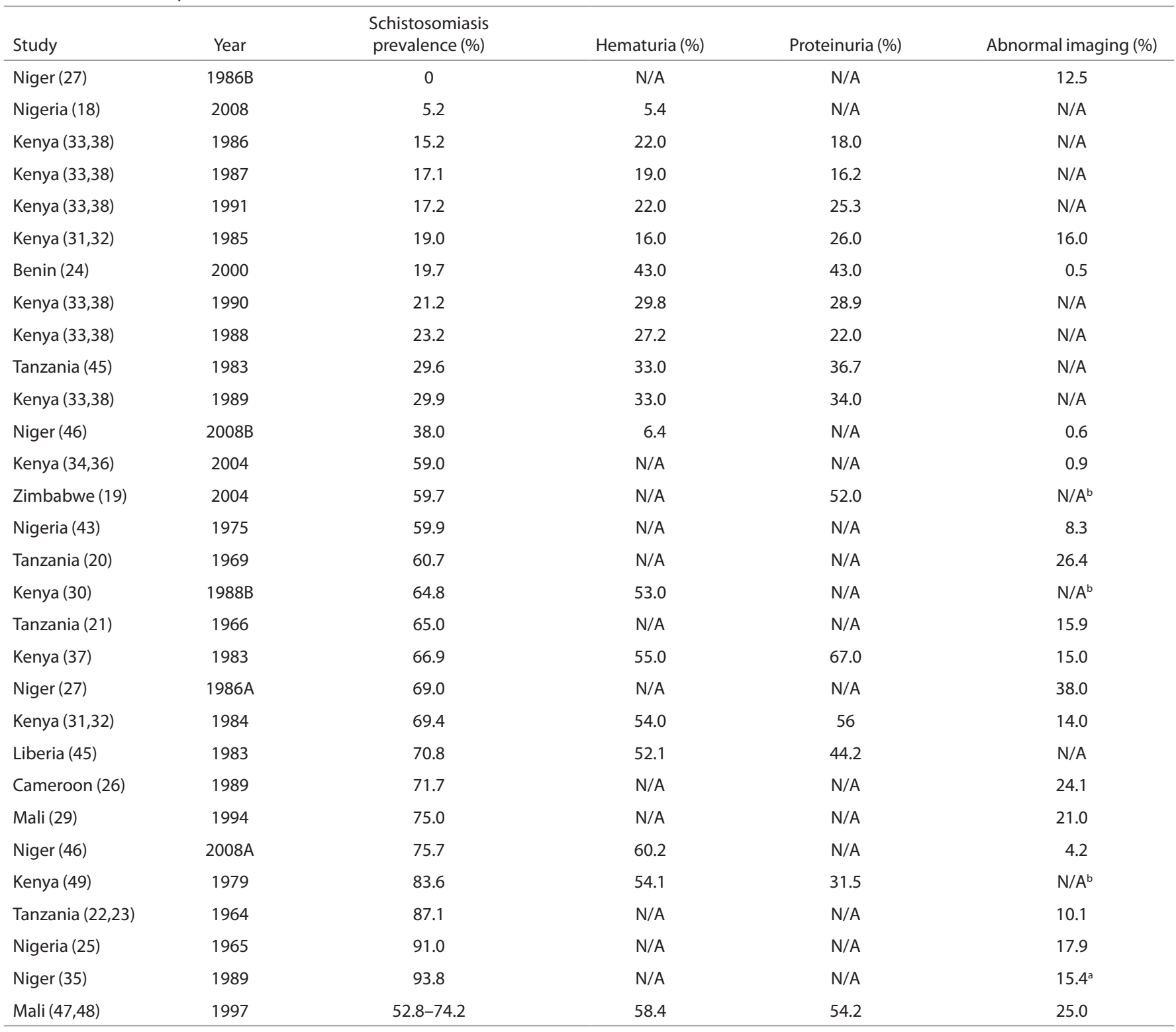

Studies have been arranged in ascending order of schistosomiasis prevalence.

N/A, not available.

aThis study did not find a positive association between schistosomiasis and ultrasound findings. ${ }^{\mathrm{b} T h e s e}$ studies reported imaging results, but only in a nonrandom subpopulation and therefore their results are excluded from analysis.

in communities where S. haematobium is common, might allow for early identification and treatment of underlying causative factors. This could prevent further damage and progression to more advanced CKD. Such a preventive measure is all the more important in a location with little to no access to renal replacement therapy.

Markers of kidney disease in children in SSA appear to be strongly associated with schistosomiasis. All studies found $S$. haematobium to be associated with hematuria and proteinuria if investigators tested for both S. haematobium and urine abnormalities. When tests were repeated at the same location in consecutive years after treatment for S. haematobium, a lower prevalence of proteinuria and hematuria was consistently discovered $(30,31,33,38,46)$. The association between
S. haematobium and urine abnormalities has been known for some time, and the presence of hematuria on dipstick has been proposed as a fairly reliable and cheap method for diagnosing S. haematobium (59). It is also known that S. haematobium can lead to proteinuria and obstructive uropathy, although the long-term progression of urinary schistosomiasis is still debated (60). Even if the hematuria, proteinuria, and obstructive uropathy from urinary schistosomiasis are reversible (after treatment for schistosomiasis), the question remains whether pediatric patients have sustained baseline kidney injury, leaving them more vulnerable to later damage from other noncommunicable diseases that are highly prevalent in low-income countries. Regular treatment of schistosomiasis in school 
children and eradication campaigns must remain a priority in areas where $S$. haematobium is highly prevalent.

Very few studies attempted to investigate the kidney function of pediatric patients, and none of these studies determined an eGFR. Among the four studies in which kidney function was assessed, SCr was the only method used. Only one study with 418 subjects tested SCr in all participants. The other three confined SCr testing to a small subgroup at higher risk for kidney dysfunction (high intensity S. haematobium infection, or concurrent proteinuria/hematuria). All of the studies reported creatinine as normal range for participating children, but they used a generous assessment for their range of normal and did not adjust their results for age, weight, or malnutrition status. Some of these values may represent a low eGFR, particularly if children were malnourished. SCr alone is a poor marker of renal function in children, especially in populations that are frequently undernourished with lower muscle mass (61). At a minimum, future studies ought to check SCr in all children and calculate an eGFR to correct for the height, age, and sex of the child. Ideally, eGFR equations should be validated in a cohort of children living in SSA rather than depending on formulae that were derived and validated using children from high-income countries.

Other well known risk factors for kidney disease are likely common among children in SSA (HIV, streptococcus, sickle cell disease, diabetes, and hypertension). None of these were systematically investigated in these studies. None of the studies, for example, intentionally included children with HIV who can have a prevalence of kidney disease as high as $31.6 \%$ $(62,63)$. More studies are needed to investigate the relative contribution of these other risk factors to the overall burden of kidney disease in children.

The quality of the studies that we identified in this systematic review was generally moderate to poor. Less than half were completed in the past 20 years. Only four measured SCr, and none calculated a eGFR or other measure of kidney function. Studies that investigated proteinuria overwhelmingly used urine dipsticks to detect urine protein concentration rather than the gold-standard albumin creatinine ratio (61). For pediatric imaging, a number of different scales were used for grading, and reporting was not consistent across studies. The time between kidney injury and kidney damage or dysfunction could not be estimated because the majority of the studies were crosssectional. These all point to the need for a large scale, intensive prospective cohort study in SSA to examine the degree of kidney disease in the pediatric population, provide a measure of the incidence and natural history of kidney disease at a population level, and clearly identify the contribution of different risk factors towards the overall burden of kidney disease.

In conclusion, this systematic review identified a substantial burden of kidney disease in community dwelling pediatric patients living in SSA. Proteinuria ranged from 2.2 to $56.0 \%$ (32.5\%), and imaging abnormalities ranged from 0.5 to $38.0 \%$ (14.8\%). Schistosomiasis was the primary risk factor identified. Screening and mass treatment for schistosomiasis ought to remain a public health priority in order to prevent cumulative renal injury in children. USS screening should be used to identify obstructive lesions. In addition, there is an urgent need for large, high quality, prospective data examining the prevalence and progression of kidney disease among children in SSA, and factors other than schistosomiasis which might be important and preventable risk factors.

\section{METHODS}

We completed a systematic review of the literature between March and June 2013 to better understand the prevalence of kidney disease in children living in SSA. An information specialist created a detailed search algorithm with input from two board certified pediatricians that combined the following search concepts: "pediatric" AND "kidney diseases" AND "sub-Saharan Africa" AND "epidemiology." We used Ovid to search both Medline and Embase, selecting controlled vocabulary appropriate to each database. We did not limit the search by study design, date of publication, or language. The search strategies for Medline and Embase are provided in Supplementary Appendix S1 online. We identified further studies by looking at the reference lists of both review articles and articles that we selected for inclusion in the final analysis. We also looked in Scopus and Web of Science for references which cited the articles that we selected for analysis. Articles in languages other than English were translated by research personnel fluent in that language.

Studies were included if they enrolled a community-dwelling population (rather than a health facility-based population), if they enrolled pediatric patients ( $<21 \mathrm{y}$ old), if they were performed among black Africans living in SSA, and if they reported an objective measure of kidney function or damage. Acceptable measures of kidney function or damage were SCr, eGFR, urine protein or urine red blood cells (either qualitative or quantitative), USS or intravenous pyelogram of the kidney and ureter, or kidney biopsy. Studies were excluded if they enrolled a combined population (e.g., adults and children, black Africans and other ethnic groups) and did not report outcomes separately for the patients of interest.

References were evaluated by two investigators independently for inclusion in analysis. First references were excluded based on title and abstract. Those which remained were reviewed in full text. Data were extracted from included articles using a standardized questionnaire. For four references $(33,34,38,42)$, data were only available in figures. For these, ImageJ64 (free share software, United States National Institutes of Health, Bethesda, MD) was used to change data into tabular format. If the same data set was reported in two publications, these were combined. If a publication reported data from distinct geographic areas or separate study years, the data sets were reported separately in the results tables.

Differences between investigators were resolved through discussion with a third investigator. Data were entered from the questionnaires into a Microsoft Excel spreadsheet. Assessment of potential selection bias was completed using a three point scale with 1 being a perfectly designed, randomly selected, representative sample of the population, 2 being an imperfectly designed, nonrandom sample of the population, and 3 being a hospital-based, highly biased sample. Measurement bias was graded on a similar three point scale with a score of 1 equivalent to an extremely accurate test of kidney function (clearance of inulin or nuclear clearance scan), 2 being a quantitative test for kidney damage or function, and 3 being a qualitative test of kidney function or damage.

\section{SUPPLEMENTARY MATERIAL}

Supplementary material is linked to the online version of the paper at http://www.nature.com/pr

\section{STATEMENT OF FINANCIAL SUPPORT}

This project was supported in part by grants from the US National Institutes of Health (NIH), National Institute of Allergy and Infectious Diseases (Al098627), the NIH Fogarty International Center (R25TW009337), and a US Agency for International Development (USAID) leadership training program. Disclosure: The authors declare no conflict of interest. 


\section{Systematic Review | Kayange etal.}

\section{REFERENCES}

1. Lozano R, Naghavi M, Foreman K, et al. Global and regional mortality from 235 causes of death for 20 age groups in 1990 and 2010: a systematic analysis for the Global Burden of Disease Study 2010. Lancet 2012;380:2095-128.

2. Stanifer JW, Jing B, Tolan S, et al. The epidemiology of chronic kidney disease in sub-Saharan Africa: a systematic review and meta-analysis. Lancet Glob Health 2014;2:e174-81.

3. The EMMES Corporation. North American Pediatric Renal Transplant Cooperative Study (NAPRTCS) 2008 Annual Report. Rockville, MD, 2008.

4. Ardissino G, Daccò V, Testa S, et al.; ItalKid Project. Epidemiology of chronic renal failure in children: data from the ItalKid project. Pediatrics 2003;111(4 Pt 1):e382-7.

5. Mong Hiep TT, Ismaili K, Collart F, et al. Clinical characteristics and outcomes of children with stage 3-5 chronic kidney disease. Pediatr Nephrol 2010;25:935-40.

6. Lipkowitz MS, Freedman BI, Langefeld CD, et al.; SK Investigators. Apolipoprotein L1 gene variants associate with hypertension-attributed nephropathy and the rate of kidney function decline in African Americans. Kidney Int 2013;83:114-20.

7. Genovese G, Friedman DJ, Ross MD, et al. Association of trypanolytic ApoL1 variants with kidney disease in African Americans. Science 2010;329:841-5.

8. Tayo BO, Kramer H, Salako BL, et al. Genetic variation in APOL1 and MYH9 genes is associated with chronic kidney disease among Nigerians. Int Urol Nephrol 2013;45:485-94.

9. Chan PY, Morris JM, Leslie GI, Kelly PJ, Gallery ED. The long-term effects of prematurity and intrauterine growth restriction on cardiovascular, renal, and metabolic function. Int J Pediatr 2010;2010:280402.

10. Zohdi V, Sutherland MR, Lim K, Gubhaju L, Zimanyi MA, Black MJ. Low Birth Weight due to Intrauterine Growth Restriction and/or Preterm Birth: Effects on Nephron Number and Long-Term Renal Health. Int J Nephrol 2012;2012:136942.

11. Ali el-TM, Abdelraheem MB, Mohamed RM, Hassan EG, Watson AR Chronic renal failure in Sudanese children: aetiology and outcomes. Pediatr Nephrol 2009;24:349-53.

12. Bhimma R, Adhikari M, Asharam K, Connolly C. The spectrum of chronic kidney disease (stages 2-5) in KwaZulu-Natal, South Africa. Pediatr Nephrol 2008;23:1841-6.

13. Anochie I, Eke F. Chronic renal failure in children: a report from Port Harcourt, Nigeria (1985-2000). Pediatr Nephrol 2003;18:692-5.

14. Asinobi AO, Ademola AD, Ogunkunle OO, Mott SA. Paediatric end-stage renal disease in a tertiary hospital in South West Nigeria. BMC Nephrol 2014;15:25.

15. Aloni MN, Nsibu CN, Meeko-Mimaniye M, Ekulu PM, Bodi JM. Acute renal failure in Congolese children: a tertiary institution experience. Acta Paediatr 2012;101:e514-8.

16. Esezobor CI, Ladapo TA, Osinaike B, Lesi FE. Paediatric acute kidney injury in a tertiary hospital in Nigeria: prevalence, causes and mortality rate. PLoS ONE 2012;7:e51229.

17. Jha V, Parameswaran S. Community-acquired acute kidney injury in tropical countries. Nat Rev Nephrol 2013;9:278-90.

18. Adesola A, Akibu O, Ademola O, Akinwale A. Haematuria in the rural primary school children in South Western Nigeria using Combi test strips. Res J Med Sci 2008;2:287-90.

19. Brouwer KC, Munatsi A, Ndhlovu PD, Wagatsuma Y, Shiff CJ. Urinary schistosomiasis in Zimbabwean school children: predictors of morbidity. Afr Health Sci 2004;4:115-8.

20. Forsyth DM. A longitudinal study of endemic urinary schistosomiasis in a small East African community. Bull World Health Organ 1969;40: $771-83$.

21. Forsyth DM, Bradley DJ. The consequences of Bilharziasis. Medical and public health importance in North-west Tanzania. Bull World Health Organ 1966;34:715-35.

22. Forsyth DM. The urological consequences of endemic bilharzial infection in schoolchildren. East Afr Med J 1964;41:567-71.
23. Forsyth DM, MacDonald G. Urological complications of endemic schistosomiasis in school-children. I. Usagara school. Trans R Soc Trop Med Hyg 1965;59:171-8.

24. Garba A, Kinde-Gazard D, Makoutodé M, et al. [Preliminary evaluation of morbidity due to S. haematobium and S. mansoni in the area of the future Adjarala Dam in Benin]. Sante 2000;10:323-8.

25. Gilles HM, Lucas A, Adeniyi-Jones C, et al. Schistosoma haematobium infection in Nigeria. II. Infection at a primary school in Ibadan. Ann Trop Med Parasitol 1965;59:441-50.

26. Gonsu-Fotsin J, Tagni-Zukam D, Moyou-Somo R, et al. [An echographic study of the urologic lesions of urinary bilharziasis in children in Barombi Kotto (Cameroon)]. Ann Radiol (Paris) 1989;32:288-92.

27. Heurtier Y, Lamothe F, Develoux M, et al. Urinary tract lesions due to Schistosoma haematobium infection assessed by ultrasonography in a community based study in Niger. Am J Trop Med Hyg 1986;35:1163-72.

28. Johansen MV, Simonsen PE, Butterworth AE, et al. A survey of Schistosoma mansoni induced kidney disease in children in an endemic area of Machakos District, Kenya. Acta Trop 1994;58:21-8.

29. Kardorff R, Traoré M, Doehring-Schwerdtfeger E, Vester U, Ehrich JH. Ultrasonography of ureteric abnormalities induced by Schistosoma haematobium infection before and after praziquantel treatment. Br J Urol 1994;74:703-9.

30. King CH, Keating CE, Muruka JF, et al. Urinary tract morbidity in schistosomiasis haematobia: associations with age and intensity of infection in an endemic area of Coast Province, Kenya. Am J Trop Med Hyg 1988;39:361-

31. King CH, Lombardi G, Lombardi C, et al. Chemotherapy-based control of schistosomiasis haematobia. II. Metrifonate vs. praziquantel in control of infection-associated morbidity. Am J Trop Med Hyg 1990;42:587-95.

32. King CH, Lombardi G, Lombardi C, et al. Chemotherapy-based control of schistosomiasis haematobia. I. Metrifonate versus praziquantel in control of intensity and prevalence of infection. Am J Trop Med Hyg 1988;39:295305.

33. King $\mathrm{CH}$, Muchiri EM, Ouma JH. Age-targeted chemotherapy for control of urinary schistosomiasis in endemic populations. Mem Inst Oswaldo Cruz 1992;87:Suppl 4:203-10.

34. King CH, Blanton RE, Muchiri EM, et al. Low heritable component of risk for infection intensity and infection-associated disease in urinary schistosomiasis among Wadigo village populations in Coast Province, Kenya. Am J Trop Med Hyg 2004;70:57-62.

35. Lamothe F, Develoux M, Devidas A, Mouchet F, Sellin B. [Echographic study of the morbidity due to urinary bilharziasis in a hyperendemic village in Niger]. Bull Soc Pathol Exot Filiales 1989;82:678-84.

36. Magak P, King CH, Ireri E, Kadzo H, Ouma JH, Muchiri EM. High prevalence of ectopic kidney in Coast Province, Kenya. Trop Med Int Health 2004;9:595-600.

37. Masaba SC, Awiti IE, Muruka JF. Morbidity in urinary schistosomiasis in relation to the intensity of infection in Kisumu, Kenya. J Trop Med Hyg 1983;86:65-6.

38. Muchiri EM, Ouma JH, King CH. Dynamics and control of Schistosoma haematobium transmission in Kenya: an overview of the Msambweni Project. Am J Trop Med Hyg 1996;55:Suppl 5:127-34.

39. Muraguri PW, McLigeyo SO, Kayima JK. Proteinuria, other selected urinary abnormalities and hypertension among teenage secondary school students in Nairobi, Kenya. East Afr Med J 1997;74:467-73.

40. Okpere AN, Anochie IC, Eke FU. Prevalence of microalbuminuria among secondary school children in Port Harcourt, Rivers State, Nigeria. Pediatr Nephrol 2010;25:1923.

41. Okpere AN, Anochie IC, Eke FU. Prevalence of microalbuminuria among secondary school children. Afr Health Sci 2012;12:140-7.

42. Oviasu E, Oviasu SV. Urinary abnormalities in asymptomatic adolescent Nigerians. West Afr J Med 1994;13:152-5.

43. Oyediran AB, Abayomi IO, Akinkugbe OO, Bohrer SP, Lucas AO. Renographic studies in vesical schistosomiasis in children. Am J Trop Med Hyg 1975;24:274-9.

44. Pugh RN, Bell DR, Gilles HM. Malumfashi Endemic Diseases Research Project, XV. The potential medical importance of bilharzia in northern 
Nigeria: a suggested rapid, cheap and effective solution for control of Schistosoma haematobium infection. Ann Trop Med Parasitol 1980;74:597-613.

45. Tanner M, Holzer B, Marti HP, Saladin B, Degrémont AA. Frequency of haematuria and proteinuria among Schistosoma haematobium infected children of two communities from Liberia and Tanzania. Acta Trop 1983;40:231-7.

46. Tohon ZB, Mainassara HB, Garba A, et al. Controlling schistosomiasis: significant decrease of anaemia prevalence one year after a single dose of praziquantel in Nigerian schoolchildren. PLoS Negl Trop Dis 2008;2: e241.

47. Traore M, Traore HA, Kardorff R, et al. The public health significance of urinary schistosomiasis as a cause of morbidity in two districts in Mali. Am J Trop Med Hyg 1998;59:407-13.

48. Vester U, Kardorff R, Traoré M, et al. Urinary tract morbidity due to Schistosoma haematobium infection in Mali. Kidney Int 1997;52:478-81.

49. Warren KS, Mahmoud AA, Muruka JF, Whittaker LR, Ouma JH, Arap Siongok TK. Schistosomiasis haematobia in coast province Kenya. Relationship between egg output and morbidity. Am J Trop Med Hyg 1979;28:864-70.

50. US Renal Data System. USRDS 2013 Annual Data Report: Atlas of Chronic Kidney Disease and End-Stage Renal Disease in the United States. Bethesda, MD: National Institutes of Health, National Institute of Diabetes and Digestive and Kidney Diseases, 2013.

51. Jones CA, McQuillan GM, Kusek JW, et al. Serum creatinine levels in the US population: third National Health and Nutrition Examination Survey. Am J Kidney Dis 1998;32:992-9.

52. Jones CA, Francis ME, Eberhardt MS, et al. Microalbuminuria in the US population: third National Health and Nutrition Examination Survey. Am J Kidney Dis 2002;39:445-59.

53. Chavers BM, Rheault MN, Foley RN. Kidney function reference values in US adolescents: National Health And Nutrition Examination Survey 19992008. Clin J Am Soc Nephrol 2011;6:1956-62.
54. Dodge WF, West EF, Smith EH, Bruce Harvey 3rd. Proteinuria and hematuria in schoolchildren: epidemiology and early natural history. J Pediatr 1976;88:327-47.

55. Hillege HL, Fidler V, Diercks GF, et al.; Prevention of Renal and Vascular End Stage Disease (PREVEND) Study Group. Urinary albumin excretion predicts cardiovascular and noncardiovascular mortality in general population. Circulation 2002;106:1777-82.

56. Lucarelli G, Ditonno P, Bettocchi C, et al. Delayed relief of ureteral obstruction is implicated in the long-term development of renal damage and arterial hypertension in patients with unilateral ureteral injury. J Urol 2013;189:960-5.

57. Warshaw BL, Edelbrock HH, Ettenger RB, et al. Progression to end-stage renal disease in children with obstructive uropathy. J Pediatr 1982;100:1837.

58. Ucero AC, Gonçalves S, Benito-Martin A, et al. Obstructive renal injury: from fluid mechanics to molecular cell biology. Open Access J Urol 2010;2:41-55.

59. King $\mathrm{CH}$, Bertsch D. Meta-analysis of urine heme dipstick diagnosis of Schistosoma haematobium infection, including low-prevalence and previously-treated populations. PLoS Negl Trop Dis 2013;7:e2431.

60. Khalaf I, Shokeir A, Shalaby M. Urologic complications of genitourinary schistosomiasis. World J Urol 2012;30:31-8.

61. Kidney Disease: Improving Global Outcomes (KIDGO) CKD Work Group. KDIGO 2012 clinical practice guideline for the evaluation and management of chronic kidney disease. Kidney Int Suppl 2013;3:1-150.

62. Esezobor CI, Iroha E, Oladipo O, et al. Kidney function of HIV-infected children in Lagos, Nigeria: using Filler's serum cystatin C-based formula. J Int AIDS Soc 2010;13:17.

63. Ikpeme EE, Ekrikpo UE, Akpan MU, Ekaidem SI. Determining the prevalence of human immunodeficiency virus-associated nephropathy (HIVAN) using proteinuria and ultrasound findings in a Nigerian paediatric HIV population. Pan Afr Med J 2012;11:13. 